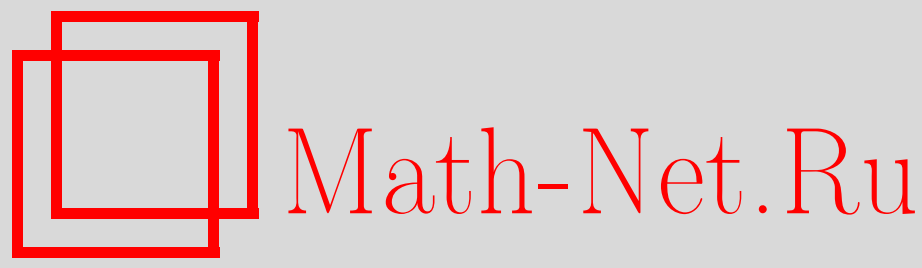

В. Н. Марачевский, Флуктуационный потенциал взаимодействия нейтрального атома с дифракционной решеткой, ТМФ, 2015, том 185, номер 1, 151-161

DOI: https://doi.org/10.4213/tmf8906

Использование Общероссийского математического портала Math-Net.Ru подразумевает, что вы прочитали и согласны с пользовательским соглашением http://www . mathnet.ru/rus/agreement

Параметры загрузки:

IP : 54.81 .137 .203

26 апреля 2023 г., 13:11:09

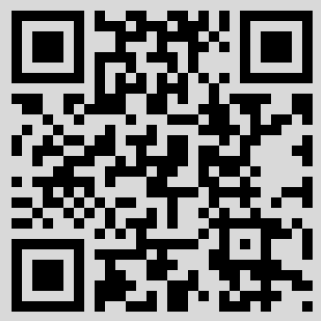




\title{
ФЛУКТУАЦИОННЫЙ ПОТЕНЦИАЛ ВЗАИМОДЕЙСТВИЯ НЕЙТРАЛЬНОГО АТОМА С ДИФРАКЦИОННОЙ РЕШЕТКОЙ
}

\begin{abstract}
Представлен теоретический формализм расчета флуктуационного потенциала взаимодействия анизотропного нейтрального атома в основном состоянии с дифракционной решеткой.
\end{abstract}

Ключевые слова: эффект Казимира-Полдера, коэффициент Рэлея.

DOI: $10.4213 / \operatorname{tmf} 8906$

\section{1. ВВЕДЕНИЕ}

Эффекты Казимира [1] и Казимира-Полдера [2] позволяют изучать флуктуационные эффекты при наличии макроскопических тел [3]-[12]. Теоретический формализм, позволяющий проводить точные расчеты сил Казимира в периодических геометриях, был предложен в работах [13], [14]. Сравнение теории и экспериментов по измерению нормальной и тангенциальной сил Казимира в периодических геометриях было впервые проведено в работах [13], [15]. В работе [13] было проведено сравнение теории и эксперимента [16] по измерению нормальной силы Казимира для геометрии с прямоугольным профилем дифракционной решетки. В работах [15] было проведено сравнение теории и экспериментов по измерению тангенциальной силы Казимира для геометрий с синусоидальными профилями поверхностей и совпадающими периодами, разделенными вакуумной щелью. В работах [15] удалось показать, что тангенциальная сила Казимира между параллельными дифракционными решетками находится в точном соответствии с результатами точной теории, а расчеты, проведенные с использованием приближения близкой силы, которое основано на теории Лифшица [17], [18] для плоскопараллельных геометрий, не согласуются с экспериментальными данными. Обзор исследований, проведенных для различных наноструктур, можно найти в работе [19].

Общий метод нахождения потенциала Казимира-Полдера в произвольной калибровке вектор-потенциалов был предложен в работе [20]. Потенциал Казимира-Полдера при наличии периодических геометрий был рассмотрен в работах [21], [22].

*Отдел теоретической физики, Санкт-Петербургский государственный университет, Санкт-Петербург, Россия. E-mail: maraval@mail.ru 
В работе [22] было проведено сравнение теории и эксперимента по рассеянию бозеэйнштейновских конденсатов атомов рубидия на дифракционной решетке при наличии дополнительного потенциала отталкивания, созданного внешним лазерным полем.

В настоящей работе представлен теоретический формализм расчета при нулевой температуре потенциала Казимира-Полдера анизотропного атома при наличии дифракционной решетки, периодической в одном пространственном направлении. В разделе 2 флуктуационный потенциал выражен через коэффициенты Рэлея [23]. Формализм расчета коэффициентов Рэлея для геометрий с прямоугольным профилем дифракционной решетки, изложенный в разделе 3 , может быть использован при расчетах потенциала Казимира-Полдера и при расчетах сил Казимира между двумя дифракционными решетками, разделенными вакуумной щелью [24].

Внутри диэлектриков предполагается выполненным условие $\mu=1$, используются единицы $\hbar=c=1$.

\section{2. ФЛУКТУАЦИОННЫЙ ПОТЕНЦИАЛ}

Рассмотрим дифракционную решетку с периодом $d$, периодическую в направлении $x$ и трансляционно-инвариантную в направлении $z$. Материал решетки определяется заданием частотной дисперсии диэлектрической проницаемости $\varepsilon(\omega)$. Рассмотрим решетку, в которой полупространство $y \leqslant 0$ заполнено диэлектриком, а периодически меняющийся профиль границы дифракционной решетки и вакуума ( $\varepsilon=1$ в вакууме) расположен при $0 \leqslant y \leqslant b$. При $y>b$ имеем $\varepsilon=1$, за исключением точки $\vec{r}_{\mathrm{A}}$, в которую помещен нейтральный атом, описывающийся атомной поляризуемостью $\alpha_{i j}(\omega)$.

Для решения задачи дифракции необходимо решить уравнения Максвелла при наличии дифракционной решетки. Электрические и магнитные поля могут быть записаны в виде

$$
\begin{aligned}
& E_{i}(x, y, z, t)=E_{i}(x, y) e^{i k_{z} z-i \omega t}, \\
& H_{i}(x, y, z, t)=H_{i}(x, y) e^{i k_{z} z-i \omega t}, \\
& E_{i}(x+d, y)=E_{i}(x, y) e^{i k_{x} d} \\
& H_{i}(x+d, y)=H_{i}(x, y) e^{i k_{x} d} .
\end{aligned}
$$

Рассмотрим плоскую электромагнитную волну, падающую (вниз) на дифракционную решетку. При $y>b$ запишем компоненты электромагнитного поля с использованием разложений Рэлея [23]:

$$
\begin{aligned}
& E_{z}(x, y)=I_{p}^{(E)} e^{i \alpha_{p} x-i \beta_{p} y}+\sum_{n=-\infty}^{+\infty} R_{n p}^{(E)} e^{i \alpha_{n} x+i \beta_{n} y} \\
& H_{z}(x, y)=I_{p}^{(H)} e^{i \alpha_{p} x-i \beta_{p} y}+\sum_{n=-\infty}^{+\infty} R_{n p}^{(H)} e^{i \alpha_{n} x+i \beta_{n} y}
\end{aligned}
$$


где

$$
\begin{array}{ll}
\alpha_{p}=k_{x}+\frac{2 \pi p}{d}, & \beta_{p}^{2}=\omega^{2}-k_{z}^{2}-\alpha_{p}^{2}, \\
\alpha_{n}=k_{x}+\frac{2 \pi n}{d}, & \beta_{n}^{2}=\omega^{2}-k_{z}^{2}-\alpha_{n}^{2},
\end{array}
$$

числа $n, p$ целые, суммирование производится по всем $n \in \mathbb{Z}$, и $-\pi / d<k_{x}<\pi / d$. Отраженная от дифракционной решетки волна определяется коэффициентами отражения Рэлея $R_{n p}^{(E)}, R_{n p}^{(H)}$. Также определим коэффициенты отражения Рэлея $R_{n p}^{E E}$, $R_{n p}^{H H}, R_{n p}^{E H}, R_{n p}^{H E}$, где второй верхний индекс соответствует индексу $\sigma=E, H$ падающей волны в $I_{p}^{(\sigma)}$ в разложениях $(1),(2)$, а первый верхний индекс соответствует электрической или магнитной отраженной волне в разложениях $(1),(2)$.

При $y \leqslant 0$ компоненты электромагнитного поля удовлетворяют разложениям

$$
\begin{aligned}
& E_{z}(x, y)=\sum_{n=-\infty}^{+\infty} T_{n p}^{(E)} e^{i \alpha_{n} x-i \beta_{n}^{(2)} y}, \\
& H_{z}(x, y)=\sum_{n=-\infty}^{+\infty} T_{n p}^{(H)} e^{i \alpha_{n} x-i \beta_{n}^{(2)} y},
\end{aligned}
$$

где

$$
\left(\beta_{n}^{(2)}\right)^{2}=\varepsilon \omega^{2}-k_{z}^{2}-\alpha_{n}^{2}
$$

Аналогично коэффициентам отражения Рэлея определим коэффициенты прохождения Рэлея $T_{n p}^{E E}, T_{n p}^{H H}, T_{n p}^{E H}, T_{n p}^{H E}$.

При $0 \leqslant y \leqslant b$ компонента электромагнитного поля $E_{z}$ определяется следующим образом:

$$
E_{z}(x, y)=\sum_{n=-\infty}^{+\infty} E_{z}^{n}(y) e^{i \alpha_{n} x}
$$

другие компоненты электромагнитного поля в области $0 \leqslant y \leqslant b$ определяются по аналогии с (5).

Для нахождения перпендикулярных оси $z$ компонент электрического и магнитного поля при $y \geqslant b$ и $y \leqslant 0$ воспользуемся формулами

$$
\begin{aligned}
& \vec{E}_{\mathrm{t}}\left(\varepsilon \omega^{2}-k_{z}^{2}\right)=i k_{z} \operatorname{grad} E_{z}-i \omega \vec{z} \times \operatorname{grad} H_{z}, \\
& \vec{H}_{\mathrm{t}}\left(\varepsilon \omega^{2}-k_{z}^{2}\right)=i k_{z} \operatorname{grad} H_{z}+i \omega \varepsilon \vec{z} \times \operatorname{grad} E_{z} .
\end{aligned}
$$

Рассмотрим следующие поляризации электромагнитной волны: $E$-поляризацию, в которой $E_{z} \neq 0, H_{z}=0$, и $H$-поляризацию, в которой $H_{z} \neq 0, E_{z}=0$. При решении задач в периодических геометриях удобен именно этот выбор поляризаций.

Компоненты электрического поля электромагнитной волны, идущей вниз, в случае $E$-поляризации равны

$$
\vec{E}_{E n}^{-}=\vec{e}_{E n}^{-} e^{i \alpha_{n} x} e^{-i \beta_{n} y} e^{i k_{z} z} e^{-i \omega t},
$$

а для $H$-поляризации

$$
\vec{E}_{H n}^{-}=\vec{e}_{H n}^{-} e^{i \alpha_{n} x} e^{-i \beta_{n} y} e^{i k_{z} z} e^{-i \omega t}
$$


здесь введены нормированные векторы поляризаций электромагнитного поля для электромагнитной волны, распространяющейся вниз,

$$
\vec{e}_{E n}^{-}=\frac{1}{C}\left(\begin{array}{c}
-\frac{k_{z} \alpha_{n}}{\omega^{2}-k_{z}^{2}} \\
\frac{k_{z} \beta_{n}}{\omega^{2}-k_{z}^{2}} \\
1
\end{array}\right), \quad \vec{e}_{H n}^{-}=\frac{1}{C}\left(\begin{array}{c}
\frac{\omega \beta_{n}}{\omega^{2}-k_{z}^{2}} \\
\frac{\omega \alpha_{n}}{\omega^{2}-k_{z}^{2}} \\
0
\end{array}\right),
$$

где $C^{2} \equiv|\vec{E}|^{2}=\omega^{2} /\left(\omega^{2}-k_{z}^{2}\right)$. Аналогично введем нормированные векторы поляризаций электромагнитного поля для электромагнитной волны, распространяющейся вверх:

$$
\vec{e}_{E n}^{+}=\frac{1}{C}\left(\begin{array}{c}
-\frac{k_{z} \alpha_{n}}{\omega^{2}-k_{z}^{2}} \\
-\frac{k_{z} \beta_{n}}{\omega^{2}-k_{z}^{2}} \\
1
\end{array}\right), \quad \vec{e}_{H n}^{+}=\frac{1}{C}\left(\begin{array}{c}
-\frac{\omega \beta_{n}}{\omega^{2}-k_{z}^{2}} \\
\frac{\omega \alpha_{n}}{\omega^{2}-k_{z}^{2}} \\
0
\end{array}\right) .
$$

Найдем тензорное произведение векторов плюс- и минус-поляризаций при евклидовом повороте $\omega \rightarrow i \omega$. Положим $K^{2}=\omega^{2}+k_{z}^{2}(K>0)$. Имеем

$$
\begin{aligned}
& \vec{e}_{E m}^{+} \otimes \vec{e}_{E n}^{-}=\frac{1}{\omega^{2}}\left(\begin{array}{ccc}
\frac{k_{z}^{2} \alpha_{m} \alpha_{n}}{K^{2}} & -\frac{i k_{z}^{2} \alpha_{m} \beta_{n}}{K^{2}} & k_{z} \alpha_{m} \\
\frac{i k_{z}^{2} \beta_{m} \alpha_{n}}{K^{2}} & \frac{k_{z}^{2} \beta_{m} \beta_{n}}{K^{2}} & i k_{z} \beta_{m} \\
k_{z} \alpha_{n} & -i k_{z} \beta_{n} & K^{2}
\end{array}\right), \\
& \vec{e}_{H m}^{+} \otimes \vec{e}_{H n}^{-}=\frac{1}{\omega^{2}}\left(\begin{array}{ccc}
-\frac{\omega^{2} \beta_{m} \beta_{n}}{K^{2}} & \frac{i \omega^{2} \beta_{m} \alpha_{n}}{K^{2}} & 0 \\
-\frac{i \omega^{2} \alpha_{m} \beta_{n}}{K^{2}} & -\frac{\omega^{2} \alpha_{m} \alpha_{n}}{K^{2}} & 0 \\
0 & 0 & 0
\end{array}\right), \\
& \vec{e}_{E m}^{+} \otimes \vec{e}_{H n}^{-}=\frac{1}{\omega^{2}}\left(\begin{array}{ccc}
\frac{\omega k_{z} \alpha_{m} \beta_{n}}{K^{2}} & -\frac{i \omega k_{z} \alpha_{m} \alpha_{n}}{K^{2}} & 0 \\
\frac{i \omega k_{z} \beta_{m} \beta_{n}}{K^{2}} & \frac{\omega k_{z} \beta_{m} \alpha_{n}}{K^{2}} & 0 \\
\vec{e}_{H m}^{+} \otimes \beta_{n} & -i \omega \alpha_{n} & 0
\end{array}\right), \\
& \left.\begin{array}{ccc}
-\frac{\omega k_{z} \beta_{m} \alpha_{n}}{K^{2}} & \frac{i \omega k_{z} \beta_{m} \beta_{n}}{K^{2}} & -\omega \beta_{m} \\
-\frac{i \omega k_{z} \alpha_{m} \alpha_{n}}{K^{2}} & -\frac{\omega k_{z} \alpha_{m} \beta_{n}}{K^{2}} & -i \omega \alpha_{m} \\
0 & 0 & 0
\end{array}\right) .
\end{aligned}
$$

Флуктуационный потенциал взаимодействия анизотропного атома с поляризуемостью $\alpha_{i j}(\omega)$, расположенного в точке с координатами $\vec{r}_{\mathrm{A}}$, и периодической структуры с периодом $d$ в направлении $x$, расположенной при $y<y_{\mathrm{A}}$, дается общим выражением [22]

$$
U\left(\vec{r}_{\mathrm{A}}\right)=\frac{1}{2 \pi} \int_{0}^{+\infty} d \zeta \zeta^{2} \alpha_{j k}(i \zeta) G_{j k}^{(1)}\left(\vec{r}_{\mathrm{A}}, \vec{r}_{\mathrm{A}}, i \zeta\right)
$$


где

$$
\begin{aligned}
G_{j k}^{(1)}\left(\vec{r}, \vec{r}^{\prime}, \omega\right)= & \frac{i}{8 \pi^{2}} \int_{-\pi / d}^{\pi / d} d k_{x} \times \\
& \times \sum_{m, n=-\infty}^{+\infty} \int_{-\infty}^{+\infty} \frac{d k_{z}}{\beta_{n}} e^{i\left(\alpha_{m} x-\alpha_{n} x^{\prime}\right)+i k_{z}\left(z-z^{\prime}\right)+i\left(\beta_{m} y+\beta_{n} y^{\prime}\right)} \times \\
& \times \sum_{\sigma, \sigma^{\prime}=E, H} R_{m n}^{\sigma \sigma^{\prime}}\left(k_{x}, k_{z}, \omega\right)\left(\vec{e}_{\sigma m}^{+} \otimes \vec{e}_{\sigma^{\prime} n}^{-}\right)_{j k} .
\end{aligned}
$$

Для дифракционной решетки, которая рассматривается в настоящей работе, функция $G_{j k}^{(1)}\left(\vec{r}, \vec{r}^{\prime}, \omega\right)$ определена при $y, y^{\prime}>b$ и зависит от коэффициентов отражения Рэлея $R_{m n}^{\sigma \sigma^{\prime}}$. Метод расчета коэффициентов Рэлея для дифракционной решетки с прямоугольным профилем сечения при $0<y<b$ приведен в следующем разделе. В формулу (12) необходимо подставить тензорные произведения (11) векторов поляризации электромагнитного поля.

\section{3. КОЭФФИЦИЕНТЫ РЭЛЕЯ}

Заменим суммирование по $n$ в бесконечных пределах в разложениях Рэлея суммированием по $n$ от $-N$ до $N$. Таким образом, в приводимые ниже в данном разделе формулы войдет целочисленный положительный параметр $N$. Обозначим символом $[G](2 N+1)$-компонентный вектор с компонентами, пронумерованными как $G_{N}, G_{N-1}, \ldots, G_{0}, \ldots, G_{-N+1}, G_{-N}$. Подчеркнем, что коэффициенты $G_{n}$ зависят от $y$ и, таким образом, правильнее было бы писать везде $G_{n}(y)$, однако мы не будем этого делать для упрощения обозначений.

Определим диагональную матрицу $\Lambda$ размера $(2 N+1) \times(2 N+1)$ :

$$
\Lambda=\operatorname{diag}\left(k_{x}+\frac{2 \pi N}{d}, k_{x}+\frac{2 \pi(N-1)}{d}, \ldots, k_{x}, \ldots, k_{x}-\frac{2 \pi(N-1)}{d}, k_{x}-\frac{2 \pi N}{d}\right) .
$$

Уравнения Максвелла для периодической в направлении $x$ геометрии при наличии трансляционной инвариантности в направлении $z$ могут быть записаны следующим образом [25]:

$$
\begin{aligned}
\frac{\partial\left[E_{z}\right]}{\partial y}-i k_{z}\left[E_{y}\right] & =i \omega\left[H_{x}\right], \\
i k_{z}\left[E_{x}\right]-i \Lambda\left[E_{z}\right] & =i \omega\left[H_{y}\right], \\
i \Lambda\left[E_{y}\right]-\frac{\partial\left[E_{x}\right]}{\partial y} & =i \omega\left[H_{z}\right], \\
\frac{\partial\left[H_{z}\right]}{\partial y}-i k_{z}\left[H_{y}\right] & =-i \omega\left[D_{x}\right], \\
i k_{z}\left[H_{x}\right]-i \Lambda\left[H_{z}\right] & =-i \omega\left[D_{y}\right], \\
i \Lambda\left[H_{y}\right]-\frac{\left[H_{x}\right]}{\partial y} & =-i \omega\left[D_{z}\right] .
\end{aligned}
$$

Используя связь $[\vec{D}]=Q[\vec{E}]$ и результаты работы $[26]$, для прямоугольного профиля дифракционной решетки имеем

$$
Q=\operatorname{diag}\left(\left\|\frac{1}{\varepsilon}\right\|^{-1},\|\varepsilon\|,\|\varepsilon\|\right)
$$


Здесь $\|\varepsilon\|-$ матрица Тёплица,

$$
\|\varepsilon\|=\left(\begin{array}{ccccc}
\varepsilon_{0} & \varepsilon_{1} & \varepsilon_{2} & \ldots & \varepsilon_{2 N} \\
\varepsilon_{-1} & \varepsilon_{0} & \varepsilon_{1} & \ddots & \vdots \\
\varepsilon_{-2} & \varepsilon_{-1} & \varepsilon_{0} & \ddots & \varepsilon_{2} \\
\vdots & \ddots & \ddots & \ddots & \varepsilon_{1} \\
\varepsilon_{-2 N} & \ldots & \varepsilon_{-2} & \varepsilon_{-1} & \varepsilon_{0}
\end{array}\right)
$$

где $\varepsilon_{n}(y)$ - коэффициенты Фурье периодической функции $\varepsilon(x+d, y)=\varepsilon(x, y)$ :

$$
\varepsilon(x, y)=\sum_{n=-\infty}^{+\infty} \exp \left(\frac{i 2 \pi n x}{d}\right) \varepsilon_{n}(y) .
$$

Из уравнения (19) следует, что

$$
\left[E_{y}\right]=\|\varepsilon\|^{-1} \frac{\Lambda\left[H_{z}\right]-k_{z}\left[H_{x}\right]}{\omega} .
$$

Это выражение необходимо подставить в уравнения (15) и (17) для исключения из них вектора $\left[E_{y}\right]$. Далее, из уравнения (16) имеем

$$
\left[H_{y}\right]=\frac{k_{z}\left[E_{x}\right]-\Lambda\left[E_{z}\right]}{\omega}
$$

и это выражение необходимо подставить в уравнения (18) и (20) для исключения из них $\left[H_{y}\right]$. В результате уравнения Максвелла можно переписать в виде

$$
\frac{\partial}{\partial y}\left(\begin{array}{l}
{\left[E_{x}\right]} \\
{\left[E_{z}\right]} \\
{\left[H_{x}\right]} \\
{\left[H_{z}\right]}
\end{array}\right)=M\left(\begin{array}{l}
{\left[E_{x}\right]} \\
{\left[E_{z}\right]} \\
{\left[H_{x}\right]} \\
{\left[H_{z}\right]}
\end{array}\right)
$$

где матрица $M$ определена следующим образом:

$$
\begin{aligned}
& M \equiv M(\omega)= \\
& =i\left(\begin{array}{cccc}
0 & 0 & -\frac{k_{z}}{\omega} \Lambda\|\varepsilon\|^{-1} & \frac{1}{\omega} \Lambda\|\varepsilon\|^{-1} \Lambda-\omega I \\
0 & 0 & -\frac{k_{z}^{2}}{\omega}\|\varepsilon\|^{-1}+\omega I & \frac{k_{z}}{\omega}\|\varepsilon\|^{-1} \Lambda \\
\frac{k_{z}}{\omega} \Lambda & \omega\|\varepsilon\|-\frac{1}{\omega} \Lambda^{2} & 0 & 0 \\
\frac{k_{z}^{2}}{\omega} I-\omega\left\|\frac{1}{\epsilon}\right\|^{-1} & -\frac{k_{z}}{\omega} \Lambda & 0 & 0
\end{array}\right) .
\end{aligned}
$$

При вычислении вакуумных эффектов энергия Казимира и энергия Казимира-Полдера выражаются через матрицы отражения, составленные из коэффициентов Рэлея на мнимых частотах. В формулу (12) для потенциала Казимира-Полдера анизотропного атома при наличии поверхности, обладающей пространственной периодичностью, матрица отражения входит на мнимых частотах, поэтому рассмотрим формализм вычисления коэффициентов отражения Рэлея $R_{m n}^{\sigma \sigma^{\prime}}\left(k_{x}, k_{z}, i \omega\right)$ на 
мнимых частотах. Матрица $M_{\mathrm{E}}$, необходимая для вычисления коэффициентов Рэлея на мнимых частотах, определяется евклидовым поворотом из матрицы (27):

$$
\begin{aligned}
& M_{\mathrm{E}} \equiv M(i \omega)= \\
& =\left(\begin{array}{cccc}
0 & 0 & -\frac{k_{z}}{\omega} \Lambda\|\varepsilon\|^{-1} & \frac{1}{\omega} \Lambda\|\varepsilon\|^{-1} \Lambda+\omega I \\
0 & 0 & -\frac{k_{z}^{2}}{\omega}\|\varepsilon\|^{-1}-\omega I & \frac{k_{z}}{\omega}\|\varepsilon\|^{-1} \Lambda \\
\frac{k_{z}}{\omega} \Lambda & -\omega\|\varepsilon\|-\frac{1}{\omega} \Lambda^{2} & 0 & 0 \\
\frac{k_{z}^{2}}{\omega} I+\omega\left\|\frac{1}{\epsilon}\right\|^{-1} & -\frac{k_{z}}{\omega} \Lambda & 0 & 0
\end{array}\right) .
\end{aligned}
$$

Далее выберем линейно независимые векторы $J^{(1)}(n)$, где $n=N, \ldots,-N$, размера $4 \times(2 N+1)$, удовлетворяющие условиям $E_{z} \neq 0, H_{z}=0$, эти векторы отвечают $E$ поляризации в падающей волне:

$$
J^{(1)}(n)=\left(\begin{array}{l}
{\left[E_{x}^{(1)}(n)\right]} \\
{\left[E_{z}^{(1)}(n)\right]} \\
{\left[H_{x}^{(1)}(n)\right]} \\
{\left[H_{z}^{(1)}(n)\right]}
\end{array}\right)
$$

где в каждом из векторов $\left[E_{x}^{(1)}(n)\right],\left[E_{z}^{(1)}(n)\right],\left[H_{x}^{(1)}(n)\right]$ единственный ненулевой элемент стоит в $(N+1-n)$-й строке,

$$
\begin{aligned}
& E_{x}^{(1)}(n)_{N+1-n}=\frac{k_{z}\left(k_{x}+2 \pi n / d\right)}{\omega^{2}+k_{z}^{2}} \exp \left(b \sqrt{\omega^{2}+k_{z}^{2}+\left(k_{x}+\frac{2 \pi n}{d}\right)^{2}}\right) \\
& E_{z}^{(1)}(n)_{N+1-n}=\exp \left(b \sqrt{\omega^{2}+k_{z}^{2}+\left(k_{x}+\frac{2 \pi n}{d}\right)^{2}}\right), \\
& H_{x}^{(1)}(n)_{N+1-n}=-\frac{\omega \sqrt{\omega^{2}+k_{z}^{2}+\left(k_{x}+2 \pi n / d\right)^{2}}}{\omega^{2}+k_{z}^{2}} \exp \left(b \sqrt{\omega^{2}+k_{z}^{2}+\left(k_{x}+\frac{2 \pi n}{d}\right)^{2}}\right),
\end{aligned}
$$

а вектор $\left[H_{z}^{(1)}(n)\right]$ нулевой. Таким образом, мы получаем $2 N+1$ линейно независимых векторов $J^{(1)}(n)$, соответствующих $n=N, \ldots,-N$.

Аналогично выберем линейно независимые векторы $J^{(2)}(n), n=N, \ldots,-N$, размера $4 \times(2 N+1)$, удовлетворяющие условиям $H_{z} \neq 0, E_{z}=0$, эти векторы отвечают $H$-поляризации в падающей волне:

$$
J^{(2)}(n)=\left(\begin{array}{l}
{\left[E_{x}^{(2)}(n)\right]} \\
{\left[E_{z}^{(2)}(n)\right]} \\
{\left[H_{x}^{(2)}(n)\right]} \\
{\left[H_{z}^{(2)}(n)\right]}
\end{array}\right)
$$


где в каждом из векторов $\left[E_{x}^{(2)}(n)\right],\left[H_{x}^{(2)}(n)\right],\left[H_{z}^{(2)}(n)\right]$ единственный ненулевой элемент стоит в $(N+1-n)$-й строке,

$$
\begin{aligned}
& \left.E_{x}^{(2)}(n)_{N+1-n}=\frac{\omega \sqrt{\omega^{2}+k_{z}^{2}+\left(k_{x}+2 \pi n / d\right)^{2}}}{\omega^{2}+k_{z}^{2}} \exp b \sqrt{\omega^{2}+k_{z}^{2}+\left(k_{x}+\frac{2 \pi n}{d}\right)^{2}}\right) \\
& H_{x}^{(2)}(n)_{N+1-n}=\frac{k_{z}\left(k_{x}+2 \pi n / d\right)}{\omega^{2}+k_{z}^{2}} \exp \left(b \sqrt{\omega^{2}+k_{z}^{2}+\left(k_{x}+\frac{2 \pi n}{d}\right)^{2}}\right) \\
& H_{z}^{(2)}(n)_{N+1-n}=\exp \left(b \sqrt{\omega^{2}+k_{z}^{2}+\left(k_{x}+\frac{2 \pi n}{d}\right)^{2}}\right)
\end{aligned}
$$

а вектор $\left[E_{z}^{(2)}(n)\right]$ нулевой. Таким образом, мы получаем $2 N+1$ линейно независимых векторов $J^{(2)}(n)$, соответствующих $n=N, \ldots,-N$.

Теперь выберем линейно независимые векторы $B^{(1)}(n), n=N, \ldots,-N$, размера $4 \times(2 N+1)$, удовлетворяющие условиям $E_{z} \neq 0, H_{z}=0$, эти векторы отвечают $E$ поляризации в отраженной волне:

$$
B^{(1)}(n)=\left(\begin{array}{l}
{\left[E_{x}^{(1)}(n)\right]} \\
{\left[E_{z}^{(1)}(n)\right]} \\
{\left[H_{x}^{(1)}(n)\right]} \\
{\left[H_{z}^{(1)}(n)\right]}
\end{array}\right)
$$

где в каждом из векторов $\left[E_{x}^{(1)}(n)\right],\left[E_{z}^{(1)}(n)\right],\left[H_{x}^{(1)}(n)\right]$ единственный ненулевой элемент стоит в $(N+1-n)$-й строке,

$$
\begin{aligned}
& E_{x}^{(1)}(n)_{N+1-n}=\frac{k_{z}\left(k_{x}+2 \pi n / d\right)}{\omega^{2}+k_{z}^{2}} \exp \left(-b \sqrt{\omega^{2}+k_{z}^{2}+\left(k_{x}+\frac{2 \pi n}{d}\right)^{2}}\right), \\
& E_{z}^{(1)}(n)_{N+1-n}=\exp \left(-b \sqrt{\omega^{2}+k_{z}^{2}+\left(k_{x}+\frac{2 \pi n}{d}\right)^{2}}\right), \\
& H_{x}^{(1)}(n)_{N+1-n}=\frac{\omega \sqrt{\omega^{2}+k_{z}^{2}+\left(k_{x}+2 \pi n / d\right)^{2}}}{\omega^{2}+k_{z}^{2}} \exp \left(-b \sqrt{\omega^{2}+k_{z}^{2}+\left(k_{x}+\frac{2 \pi n}{d}\right)^{2}}\right),
\end{aligned}
$$

а вектор $\left[H_{z}^{(1)}(n)\right]$ нулевой. Таким образом, получаем $2 N+1$ линейно независимых векторов $B^{(1)}(n)$, соответствующих $n=N, \ldots,-N$.

Далее выберем линейно независимые векторы $B^{(2)}(n), n=-N, \ldots, N$, размера $4 \times(2 N+1)$, удовлетворяющие условиям $H_{z} \neq 0, E_{z}=0$, эти векторы отвечают $H$ поляризации в отраженной волне:

$$
B^{(2)}(n)=\left(\begin{array}{l}
{\left[E_{x}^{(2)}(n)\right]} \\
{\left[E_{z}^{(2)}(n)\right]} \\
{\left[H_{x}^{(2)}(n)\right]} \\
{\left[H_{z}^{(2)}(n)\right]}
\end{array}\right)
$$


где в каждом из векторов $\left[E_{x}^{(2)}(n)\right],\left[H_{x}^{(2)}(n)\right],\left[H_{z}^{(2)}(n)\right]$ единственный ненулевой элемент стоит в $(N+1-n)$-й строке,

$$
\begin{aligned}
& E_{x}^{(2)}(n)_{N+1-n}=-\frac{\omega \sqrt{\omega^{2}+k_{z}^{2}+\left(k_{x}+2 \pi n / d\right)^{2}}}{\omega^{2}+k_{z}^{2}} \exp \left(-b \sqrt{\omega^{2}+k_{z}^{2}+\left(k_{x}+\frac{2 \pi n}{d}\right)^{2}}\right) \\
& H_{x}^{(2)}(n)_{N+1-n}=\frac{k_{z}\left(k_{x}+2 \pi n / d\right)}{\omega^{2}+k_{z}^{2}} \exp \left(-b \sqrt{\omega^{2}+k_{z}^{2}+\left(k_{x}+\frac{2 \pi n}{d}\right)^{2}}\right) \\
& H_{z}^{(2)}(n)_{N+1-n}=\exp \left(-b \sqrt{\omega^{2}+k_{z}^{2}+\left(k_{x}+\frac{2 \pi n}{d}\right)^{2}}\right)
\end{aligned}
$$

а вектор $\left[E_{z}^{(2)}(n)\right]$ нулевой . Таким образом, получаем $2 N+1$ линейно независимых векторов $B^{(2)}(n)$, соответствующих $n=N, \ldots,-N$.

Выберем $2 N+1$ линейно независимых векторов $V^{(1)}(n), n=N, \ldots,-N$, размера $4 \times(2 N+1)$, удовлетворяющих условиям $E_{z} \neq 0, H_{z}=0$, эти векторы отвечают $E$ поляризации в прошедшей волне:

$$
V^{(1)}(n)=\left(\begin{array}{l}
{\left[E_{x}^{(1)}(n)\right]} \\
{\left[E_{z}^{(1)}(n)\right]} \\
{\left[H_{x}^{(1)}(n)\right]} \\
{\left[H_{z}^{(1)}(n)\right]}
\end{array}\right)
$$

где в каждом из векторов $\left[E_{x}^{(1)}(n)\right],\left[E_{z}^{(1)}(n)\right],\left[H_{x}^{(1)}(n)\right]$ единственный ненулевой элемент стоит в $(N+1-n)$-й строке,

$$
\begin{aligned}
& E_{x}^{(1)}(n)_{N+1-n}=\frac{k_{z}\left(k_{x}+2 \pi n / d\right)}{\varepsilon \omega^{2}+k_{z}^{2}}, \\
& E_{z}^{(1)}(n)_{N+1-n}=1, \\
& H_{x}^{(1)}(n)_{N+1-n}=-\frac{\omega \varepsilon \sqrt{\omega^{2} \varepsilon+k_{z}^{2}+\left(k_{x}+2 \pi n / d\right)^{2}}}{\varepsilon \omega^{2}+k_{z}^{2}},
\end{aligned}
$$

а вектор $\left[H_{z}^{(1)}(n)\right]$ нулевой. Таким образом, получаем $2 N+1$ линейно независимых векторов $V^{(1)}(n)$, соответствующих $n=N, \ldots,-N$. Уточним, случай $n=N$ соответствует вектору $\left[E_{z}^{(1)}(N)\right]$ с единственной ненулевой первой компонентой, равной единице, а случай $n=-N$ соответствует вектору $\left[E_{z}^{(1)}(-N)\right]$ с единственной ненулевой последней компонентой, равной единице.

Наконец, выберем $2 N+1$ линейно независимых векторов $V^{(2)}(n), n=N, \ldots,-N$, размера $4 \times(2 N+1)$, удовлетворяющих условиям $H_{z} \neq 0, E_{z}=0$, эти векторы отвечают $H$-поляризации в прошедшей волне:

$$
V^{(2)}(n)=\left(\begin{array}{l}
{\left[E_{x}^{(2)}(n)\right]} \\
{\left[E_{z}^{(2)}(n)\right]} \\
{\left[H_{x}^{(2)}(n)\right]} \\
{\left[H_{z}^{(2)}(n)\right]}
\end{array}\right)
$$


где в каждом из векторов $\left[E_{x}^{(2)}(n)\right],\left[H_{x}^{(2)}(n)\right],\left[H_{z}^{(2)}(n)\right]$ единственный ненулевой элемент стоит в $(N+1-n)$-й строке,

$$
\begin{aligned}
& E_{x}^{(2)}(n)_{N+1-n}=\frac{\omega \sqrt{\omega^{2} \varepsilon+k_{z}^{2}+\left(k_{x}+2 \pi n / d\right)^{2}}}{\varepsilon \omega^{2}+k_{z}^{2}}, \\
& H_{x}^{(2)}(n)_{N+1-n}=\frac{k_{z}\left(k_{x}+2 \pi n / d\right)}{\varepsilon \omega^{2}+k_{z}^{2}}, \\
& H_{z}^{(2)}(n)_{N+1-n}=1,
\end{aligned}
$$

а вектор $\left[E_{z}^{(1)}(n)\right]$ нулевой. Таким образом, получаем $2 N+1$ линейно независимых векторов $V^{(2)}(n)$, соответствующих $n=N, \ldots,-N$.

Теперь можно найти коэффициенты Рэлея для отражения от дифракционной решетки. Для этого необходимо проинтегрировать систему линейных уравнений первого порядка (26) с матрицей $(28)$ в интервале $y \in[0, b]$. В качестве начальных условий при $y=0$ берутся векторы $V^{(1)}(n)$ и $V^{(2)}(n)$. Определим $U^{(1)}(n)$ - результат решения системы уравнений $(26)$ при $y=b$ с начальным заданным вектором $V^{(1)}(n)$ при $y=0$. Аналогично определим $U^{(2)}(n)$ - результат решения системы уравнений (26) при $y=b$ с заданным вектором $V^{(2)}(n)$ при $y=0$.

При $y=b$ нужно записать условия непрерывности для всех $4 \times(2 N+1)$ фурье-компонент электромагнитного поля, тангенциальных к плоскости $y=b$, так как поля $E_{x}, E_{z}, H_{x}, H_{z}$ должны быть непрерывны при $y=b$. Из этого условия и определяются все необходимые коэффициенты Рэлея.

В результате получаем две системы линейных уравнений, из которых находятся коэффициенты отражения Рэлея $R$ и коэффициенты прохождения Рэлея $T$ :

$$
\begin{aligned}
& \sum_{n=-N}^{N}\left(-T_{n p}^{E E} U^{(1)}(n)-T_{n p}^{H E} U^{(2)}(n)+R_{n p}^{E E} B^{(1)}(n)+R_{n p}^{H E} B^{(2)}(n)\right)=-J^{(1)}(p), \\
& \sum_{n=-N}^{N}\left(-T_{n p}^{E H} U^{(1)}(n)-T_{n p}^{H H} U^{(2)}(n)+R_{n p}^{E H} B^{(1)}(n)+R_{n p}^{H H} B^{(2)}(n)\right)=-J^{(2)}(p),
\end{aligned}
$$

где $p=N, \ldots,-N$. Матрица каждой из этих систем уравнений является квадратной и имеет размер $4(2 N+1) \times 4(2 N+1)$. Подчеркнем, получающиеся коэффициенты отражения и прохождения определены на мнимых частотах.

\section{4. ЗАКЛЮЧЕНИЕ}

В работе представлен теоретический формализм расчета флуктуационного потенциала взаимодействия анизотропного нейтрального атома в основном состоянии с дифракционной решеткой, периодической в заданном пространственном направлении и трансляционно-инвариантной в перпендикулярном направлении.

Данное исследование было завершено к 90-летию со дня рождения Юрия Викторовича Новожилова, научного руководителя и замечательного человека.

Благодарности. Работа частично поддержана Санкт-Петербургским государственным университетом (гранты № 11.38.237.2015 и 11.38.660.2013). 


\section{Список литературы}

[1] H. B. G. Casimir, Proc. K. Ned. Akad. Wetensc., 51 (1948), 793-795.

[2] H. B. G. Casimir, D. Polder, Phys.Rev, 73:4 (1948), 360-372.

[3] Ю. С. Бараш, В. Л. Гинзбург, УФН, 116:1 (1975), 5-40; 143:2 (1984), 345-389.

[4] G. Plunien, B. Müller, W. Greiner, Phys. Rep., 134:2-3 (1986), 87-193.

[5] K. A. Milton, J. Phys. A: Math. Gen., 37:38 (2004), R209-R277, arXiv: hep-th/0406024.

[6] R. L. Jaffe, Phys. Rev. D, 72:2 (2005), 021301, 5 pp., arXiv: hep-th/0503158.

[7] V. N. Marachevsky, J. Phys. A: Math. Theor., 45:37 (2012), 374021, 17 pp.

[8] S. Scheel, S. Y. Buhmann, Acta Phys. Slovaca, 58:5 (2008), 675-809.

[9] S. Y. Buhmann, Dispersion Forces, Springer, Berlin, Heidelberg, 2012.

[10] D. Fursaev, D. Vassilevich, Operators, Geometry and Quanta. Methods of Spectral Geometry in Quantum Field Theory, Springer-Verlag, Dordrecht, 2011.

[11] Е. М. Сантанжело, ТМФ, 131:1 (2002), 98-117.

[12] M. Bordag, I. Pirozhenko, Phys. Rev. D, 81:8 (2010), 085023, 12 pp., arXiv: 0912.4047.

[13] A. Lambrecht, V. N. Marachevsky, Phys. Rev. Lett., 101:16 (2008), 160403.

[14] A. Lambrecht, V. N. Marachevsky, Internat. J. Modern Phys. A, 24:8-9 (2009), 1789-1795.

[15] H.-C. Chiu, G. L. Klimchitskaya, V. N. Marachevsky, V. M. Mostepanenko, U. Mohideen, Phys. Rev. B, 80:12 (2009), 121402, 4 pp.; 81:11 (2010), 115417, 20 pp.

[16] H. B. Chan, Y. Bao, J. Zou, R. A. Cirelli, F. Klemens, W. M. Mansfield, C.S. Pai, Phys. Rev. Lett., 101:3 (2008), 030401, 4 pp.

[17] Е. М. Лифшиц, ЖЭТФ, 29:1 (1955), 94-112.

[18] V. V. Nesterenko, I. G. Pirozhenko, Phys. Rev. A, 86:5 (2012), 052503, 13 c., arXiv: 1112.2599 .

[19] A. W. Rodriguez, F. Capasso, S. G. Johnson, Nature Photonics, 5:4 (2011), 211-221.

[20] V. N. Marachevsky, Yu. M. Pis'mak, Phys. Rev. D, 81:6 (2010), 065005, 6 pp.

[21] A. M. Contreras-Reyes, R. Guérout, P. A. M. Neto, D. A. R. Dalvit, A. Lambrecht, S. Reynaud, Phys. Rev. A, 82:5 (2010), 052517, 6 pp., arXiv: 1010.0170.

[22] H. Bender, C. Stehle, C. Zimmermann, S. Slama, J. Fiedler, S. Scheel, S. Y. Buhmann, V. N. Marachevsky, Phys. Rev. X, 4:1 (2014), 011029, 10 pp.

[23] O. M. Rayleigh, Proc. Roy. Soc. A, 79:532 (1907), 399-416.

[24] В. Н. Марачевский, ТМФ, 176:1 (2013), 117-126.

[25] D. R. Maystre (ed.), Selected Papers on Diffraction Gratings, SPIE, Bellingham, 1993; R. Petit (ed.), Electromagnetic Theory of Gratings, Springer, Berlin, 1980; M. C. Hutley, Diffraction Gratings, Academic Press, London, 1982; E. G. Loewen, E. Popov, Diffraction Gratings and Applications, Marcel Dekker, New York, 1997.

[26] L. Li, J. Opt. Soc. Amer. A, 13:9 (1996), 1870-1876. 